\title{
Self-Perceived Halitosis and Associated Oral Hygiene Practices among Dental Students of National Ribat University Khartoum/Sudan
}

\author{
Zeinab Ahmed Abu Sabeib ${ }^{* *}$ and Yosra Abdelfatah Hassan Ali ${ }^{2}$ \\ 'Princess Nourah bint Abdulrahman University, College of Nursing, Riyadh, Kingdom of Saudi Arabia; zaabusa- \\ beib@pnu.edu.sa \\ 2Faculty of Dentistry, National Ribat University, Khartoum, Sudan; yosra.ali87@gmail.com
}

\begin{abstract}
Background: Halitosis is the unpleasant odor that comes from the mouth; conditions intra-orally such as bad oral hygiene, poor restorations, gingival inflammation, and periodontal diseases contribute to halitosis. Successful treatment is connected to recognizing the related factors and treat them effectively. Objectives: The purpose of this study is to evaluate the selfperceived halitosis prevalence, and associated oral hygiene practices among dental students from National Ribat University Khartoum/Sudan. Methods: A cross-sectional study has been carried out. A structured self-administered questionnaire was given to undergraduate healthy students. Chi-square was used to test differences between frequencies and to assess the correlation between halitosis self-perception and oral hygiene measures. Results: The study was carried out on 150 healthy dental students; 94 females and 56 males. Self-perceived malodor is more common among males $(\mathrm{P}=0.04)$. The use of commercial productsto alleviate bad breath, and mouthwash are more widespread among females $(\mathrm{P}=0.003$, $\mathrm{P}=$.002). Males reported a significantly higher prevalent malodor among relatives and more repeated bad breath when they did the hand on mouth test $(\mathrm{P}=0.02)$. Usage of toothpicks is more among males $(\mathrm{P}=0.02)$.Self-perceived bad breath significantly related to practices of oral hygiene involving regular brushing $(\mathrm{P}=0.01)$, flossing $(\mathrm{P}=0.000)$, mouthwash and toothpicks usage $(\mathrm{P}=0.000)$. Conclusion: Undergraduate students' self-perceived malodor prevalence is comparable to the other studies. The knowledge about halitosis should be fostered, and the dental professionals should improve the health understanding in the society.
\end{abstract}

Keywords: Dental Students, Halitosis, Oral Hygiene, Oral Malodor, Sudan

\section{Introduction}

Halitosis is the unpleasant odor that comes from the mouth ${ }^{1}$.Malodor that have its origin from the mouth is found in $80-90 \%$ of cases 2 . Conditions intra-orally such as bad oral hygiene, coated tongue, sites of extraction, necrosis, caries, impaction of food, prosthetic appliances poor restorations, gingival inflammation, and periodontal diseases contribute to halitosis ${ }^{3-5}$. The non-oral malodor causes include upper respiratory tract and gastrointestinal tract disorders and specific drugs ${ }^{\underline{6}}$.
Volatile sulfur compounds (VSCs) are foul smelling bacterial chemical end-products. Non-sulfur containing compounds have a restricted relation oral malodor ${ }^{\mathrm{T}}$. VSCs such as methyl mercaptan, hydrogen sulfide, dimethyl disulfide, and dimethyl sulfide make more than $90 \%$ of the foul odors ${ }^{\text {? }}$.

Patients having periodontal diseases have increased number of leukocytes in the saliva and the level of generated methyl mercaptan are correspond with exudate of the gingiva, bleeding on probing, and depth of the pocket.

${ }^{*}$ Author for correspondence 
VSCs may participate in the periodontal disease advancement ${ }^{9}$.

McNamara and colleagues stated that the change in incubated saliva microbial flora from a predominately gram-positive to a predominately gram-negative anaerobic flora is consistent with the formation of foul odors from $\frac{10}{}$.

Halitosis is categorized into genuine, halitophobia halitosis, and pseudo-halitosis ${ }^{(11)}$;the genuine halitosis is intensive and over the appropriate social level, if the person still suffers from oral malodor after genuine malodor treatment, the condition is diagnosed as halitophobia. If the malodor is not recognized by other people but the person constantly complains of it, it is diagnosis is pseudo-halitosis ${ }^{11}$.

Effective treatment is linked to recognizing the contributing elements and treat them efficiently. Patient will go through evaluation which involves evaluation of the medical history, oral hygiene, and dental health. After the reasons of the existing dental health problem has been established, the dentist will construct a plan that include various treatments, counting on the patient'sdemands ${ }^{16}$.

The optimum aim of treatment for oral malodor should be targeted at microorganisms and correlated substrates removal or reduction with better oral hygiene and control of periodontal disorders. When the plaque and microorganisms' are controlled, their VSCs making abilities are decreased ${ }^{16}$.

The objective of the study is to evaluate the self-perceived halitosis prevalence, and associated oral hygiene practices among dental students from National Ribat University Khartoum/Sudan.

\section{Materials and Methods}

A cross-sectional study has been carried out in College of Dentistry/ National Ribat University in Khartoum State/ Sudan. A structured self-administered questionnaire was given to undergraduate healthy students aged20-24 years old.

The questionnaire was developed by reviewing the literature to investigate self-perception of halitosis and other factors related to it including the timing of oral halitosis, treatment done for the oral malodor, gingival bleeding and caries, dryness of oral cavity, smoking, tea and coffee drinking routine, coated tongue, and oral hygiene practices.
A pilot survey was done to assess the questionnaire's validity, the feasibility of the study and gain information to promote the study.

Ethical approval was given from the research committee at the National Ribat University - Faculty of Dentistry, subsequently verbal consent was obtained from the participants after describing the aim of the study.

The analysis of the data was done using the SPSS version 20. Analysis for frequency distribution was done. Chi-square was used to assess differences between frequencies and to investigate the correlation between oral malodor self-perception and practices of oral hygiene.

\section{Results}

The study was done on 150 healthy dental students, 94 females and 56 males, the response rate was $97 \%$. About $7.1 \%$ of males $(\mathrm{P}=0.04)$ and $1.51 \%$ of females were diagnosed having halitosis by a dentist and $3.6 \%$ of the males and $1.1 \%$ of the females were diagnosed by physicians smelling their exhaled air odor. Almost $3.6 \%$ of males and $1.1 \%$ of females' students have been given treatment from their physicians.

The use of commercial products to alleviate oral bad breath is significantly $(\mathrm{P}=0.003)$ more among females (53.2\%) than males(28.6\%).The use of traditional medications, such as clove oil, is nearly equal between males and females; $12.5 \%$ and $12.8 \%$ of males and females respectively.

About $19.6 \%$ of males and $7.4 \%$ of females stated having present malodor among relatives $(\mathrm{P}=0.02)$. There is a significant difference in prevalence of self-perceived malodor between males and females $(48.2 \%$ and $31.9 \%$, respectively, $\mathrm{P}=0.04$ ). About $23.1 \%$ of males and $18.1 \%$ of females had frequent bad breath on hand on mouth assessment $(\mathrm{P}=0.02)$. Malodor is more prevalent after wake up time (69.6\% for males and $63.8 \%$ for females).

In $41.1 \%$ of the males' and $28.7 \%$ of the females' participants, malodor is present with thirst or hunger. Less than thirteen percent of the students are having malodor during morning, afternoon or throughout the day.

Differences in oral hygiene practices was seen, almost $91.1 \%$ and $96 \%$ of the males and females respectively brush their teeth in regular basis. About $17.9 \%$ of the males and $30.9 \%$ of females floss their teeth, while mouthwash is used by about $19.6 \%$ males and significantly more by $(44.7 \%)$ females $(\mathrm{P}=0.002)$. Toothpicks 
usage is significantly $(\mathrm{P}<0.02)$ more among males $(41.1 \%)$ when compared with females (23.4\%).

There had been significant variance in use of miswak (Twig made from the Salvadora persica tree; called Arak), about $12 \%$ of the males use miswak against $2.1 \%$ of females $(\mathrm{P}=0.01)$.

Almost $55.4 \%$ of the males and about $47.9 \%$ of females have caries, while twenty-five percent of the males and $12.8 \%$ of females have gingival bleeding. A considerable percentage of the males $(26.8 \%)$ and females $(20.2 \%)$ of the females reported having a coated tongue.

Smoking among males and females had a significant variance $(\mathrm{P}<0.001)$; about $26.8 \%$ of males and $2.1 \%$ of females were smokers. Mouth dryness is higher in females than males; $17 \%$ and $12.5 \%$, respectively. Consumption of tea and coffee is significantly more common among males $83.9 \%$ than females $67 \%(\mathrm{P}<0.02)$.

Self-perceived bad breath significantly related to oral hygiene practices involving regular brushing $(\mathrm{P}=.01)$, flossing $(\mathrm{P}=.000)$. Mouthwash $(\mathrm{P}=.000)$ and toothpicks $(\mathrm{P}=0.000)$ usage.

\section{Discussion}

Diagnosis of the actual halitosis reason is hard sometimes, in many studies, including this study, the evaluation of malodor depends on the person's self-perception. Selfperception of halitosis relies on many factors and not consistent among people ${ }^{2,17}$, regardless its limitations, this method has been the most widely used one for halitosis evaluation ${ }^{13}$.

Few students were diagnosed by dentists $(\mathrm{P}=0.04)$, who gave treatment for less than half of them, on the other hand, a great proportion of the students haven't visited physicians for diagnosis. Diagnosis of oral malodor is mainly done by the dentist, but still the percentage of people receive treatment for it is minimal.

However, a study in United States' dentists revealed that $41 \%$ of the dentists had six or more patients per week with bad mouth odor ${ }^{18}$

In this study, the presence of self-perceived malodor was less among the females and the use of commercial products to alleviate bad breath odor was significantly $(\mathrm{P}=0.003)$ more present among females. Causes for this could be related to that females usually care about their oral hygiene and behaving appropriately ${ }^{\underline{6}}$.
In the developed world it was rated that $8-50 \%$ of the people have self-perceived oral malodor $\frac{19}{}$, Ten to thirty percent of the United States society suffer regularly from oral malodor ${ }^{20}$. Another a study in Japan showed that $24 \%$ of the assessed population suffered from bad breath ${ }^{21}$.In a French study, 22\% addressed having halitosis $\underline{22}$, while a study in Sweden stated that only $2.4 \%$ of the population participated in the study had bad breath odor ${ }^{23}$.

When hand on mouth test was done more males smelled bad breath compared to females $(\mathrm{P}=.02)$. However, these findings cannot be verified, as the selfevaluation is subjective $\mathrm{e}^{17}$.

There is agreement that after waking up most people have bad oral malodor ${ }^{24}$, considerable proportion of both males and females participated in the study, reported malodor after waking up and when being thirsty or hungry. Sleeping and being hungry or thirsty reduce the flow of saliva and cause halitosis. However, under these circumstances the halitosis disappears after eating or drinking, and should not be considered as true halitosis. Less than $13 \%$ of the participants reported halitosis in the morning, afternoon or throughout the day, these results are within the range with a study done in Saudi Arabia ${ }^{25}$.

Insufficiency in oral hygiene practices, including brushing, mouthwashes and floss usage, has been implicated in bad breath ${ }^{25}$. In this study, the brushing is more frequent in females (96\%) than in males $(91.1 \%)$. Furthermore, females used mouthwash significantly more than the males $(\mathrm{P}=.002)$. Also, females floss their teeth more frequent, this reveal better attitude among females than among males. Porter S and Scully C, found that teeth brushing among male students was $49-79.5 \% \frac{19}{}$.

Toothpicks usage is more among males in the present study $(\mathrm{P}=.02)$. Miswak, the traditional chewing stick that is commonly used in the Middle East, particularly in Saudi Arabia, has an antimicrobial action and is as effective as toothbrush plaque removal and gingival inflammationreduction ${ }^{25}$. Its use among Sudanese is infrequent; particularly by females and its effectiveness in prevention and treatment of periodontal diseases is unrealizable by a large percentage of the population. Therefore, people should be educated for its benefits. In this study, it's use among males significantly $(\mathrm{P}=0.01)$ more than females.

In line with other studies, this study showed a relation between periodontal clinical measures and oral malodor. Another study could not find a relation between halitosis 
grading and clinical periodontal measures, such as plaque index, gingival index, gingival bleeding index, and the depth of the pocket ${ }^{26}$. The relation between those parameters and oral hygiene practices on one hand and halitosis on the other needs more examination.

In the current study, dental caries is higher in males than females as well as the gingival bleeding, about half of the students have dental caries. Moreover, the prevalence of gingival bleeding is almost twice as high among males comparing to females, caries and periodontal diseases are factors related to the halitosis $\underline{19}, \underline{23}$.

In younger individuals, the presence of halitosis in the absence of periodontal disease has been assign to tongue coating $^{27}$ and it is an involve development in the halitosis etiology as a result of fermentation of bacteria and presence of VSCs and others $s^{2-29}$

In the present study, the coated tongue is reported by $25 \%$ of the males which was higher than the females, tongue cleaning aids in decreasing bacteria and bad breath $^{\underline{30}}$.

Smoking has been identified as a cause of bad breath ${ }^{(28)}$. The stereotype in the Sudanese community females are non-smokers, that's why it was more prevalent among the males $(\mathrm{P}=.000)$. Smoking showed no relation perceiving malodor and many people smoke to overcome their halitosis, that's maybe connected to the Olfactory sensitivity dropping caused by smoking $\frac{1}{}$. However, individuals with halitosis should be helped to quit smoking ${ }^{6}$.

Mouth dryness reduces the cleansing effect and causes tongue coating and bad breath, mouth dryness is more present among females, in another study in the United States the dry mouth and bad breath remained persist in 10 to $30 \%$ of the population ${ }^{20}$.

The number of males drinking tea and coffee significantly more than the females $(\mathrm{P}=.02)$. Knowledge available about the effect of those drinks in halitosis islittle ${ }^{31}$; some studies reported a relation between them and the reduction in specific oral microorganisms $s^{31}$.

\section{Conclusion}

The results of this study indicate that the undergraduate students' self-perceived malodor prevalence is within the range of other studies. However, to confirm the halitosis presence, these findings need to be augmented by objective examination.

The knowledge about halitosis as an individual entity should be fostered and dental professionals have to be role models for the society, they should embrace the populations' health understanding. The self-reported information needs to be assessed as there are accuracy concerns about them.

The study recommends more examination using the standard methods available to investigate the halitosis problem. Subjective, self-reported information is needed to be augmented by objective methods. There should be aid and assistance for the students to stop smoking and be free from bad breath.

\section{References}

1. Tangerman A. Halitosis in medicine: a review. INT DENT J. 2002; 52(S5P1): 201-206. https://doi.org/10.1002/j.1875595X.2002.tb00925.x.

2. Tonzetich J. Production and origin of oral malodor: a review of mechanisms and methods of analysis. J. Periodontol. 1977; 48(1): 13-20. https://doi.org/10.1902/ jop.1977.48.1.13.

3. Spielman A, Bivona P, Rifkin B. Halitosis. A common oral problem. N Y State Dent J. 1996; 62(10): 36-42.

4. Liu XN, Shinada K, Chen XC, Zhang BX, Yaegaki K, Kawaguchi Y. Oral malodor-related parameters in the Chinese general population. J. Clin. Periodontol. 2006;33(1): 31-36. https://doi.org/10.1111/j.1600-051X.2005.00862.x.

5. Yaegaki K, Sanada K. Biochemical and clinical factors influencing oral malodor in periodontal patients. J. Periodontol. 1992; 63(9): 783-789. https://doi.org/10.1902/ jop.1992.63.9.783.

6. Rosenberg M. Clinical assessment of bad breath: current concepts. J Am. Dent. Assoc. 1996; 127(4): 475-482. https://doi.org/10.14219/jada.archive.1996.0239.

7. Feller L, Blignaut E. Halitosis: a review. S. Afr. Dent. J. (SADJ). 2005; 60(1): 17-19.

8. Tangerman A, Winkel EG. Intra-and extra-oral halitosis: finding of a new form of extra-oral blood-borne halitosis caused by dimethyl sulphide. J. Clin. Periodontol. 2007; 34(9): 748-755. https://doi.org/10.1111/j.1600051X.2007.01116.x.

9. $\mathrm{Ng} \mathrm{W}$, Tonzetich J. Effect of hydrogen sulfide and methyl mercaptan on the permeability of oral mucosa. J. Dent. Res. 1984; 63(7): 994-997. https://doi.org/10.1177/0022034584 0630071701.

10. Preti G, Clark L, Cowart BJ, Feldman RS, Lowry LD, Weber E. Non-oral etiologies of oral malodor and altered chemosensation. J. Periodontol. 1992; 63(9): 790-796. https://doi. org/10.1902/jop.1992.63.9.790.

11. Yaegaki K, Coil JM. Examination, classification, and treatment of halitosis; clinical perspectives. J. Can. Dent. Assoc. 2000; 66(5): 257-261. 
12. Rosenberg M, Kozlovsky A, Wind Y, Mindel E. Selfassessment of oral malodor 1 year following initial consultation. Quintessence international. 1999; 30(5): 324-327.

13. Oral malodor [internet]. https://www.ncbi.nlm.nih.gov/ pubmed/12636125. Date accessed: 02/2003.

14. Durham T, Malloy T, Hodges E. Halitosis: knowing when' bad breath' signals systemic disease. Geriatrics (Basel, Switzerland). 1993; 48(8) 55-59.

15. Moriyama T. Clinical study of the correlation between bad breath and subgingival microflora. Shika gakuho Dental science reports. 1989; 89(9): 1425-1439.

16. DE BOEVER EH, Loesche WJ. Assessing the contribution of anaerobic microflora of the tongue to oral malodor. J. Am. Dent. Assoc. 1995; 126(10): 1384-1393. https://doi. org/10.14219/jada.archive.1995.0049.

17. Rosenberg M, Kozlovsky A, Gelernter I, Cherniak O, Gabbay J, Baht R. Self-estimation of oral malodor. J. Dent. Res. 1995; 74(9): 1577-1582. https://doi.org/10.1177/00220 345950740091201.

18. Loesche WJ. The effects of antimicrobial mouthrinses on oral malodor and their status relative to US Food and Drug Administration regulations. Quintessence international. 1999; 30(5): 311-318.

19. Porter S, Scully C. Oral malodour (halitosis). Br. Med. J. 2006; 333(7569): 632-635. https://doi.org/10.1136/ bmj.38954.631968.AE.

20. Meskin LH. A breath of fresh air. J. Am. Dent. Assoc. 1996; 127(9): 1282-1284. https://doi.org/10.14219/jada. archive.1996.0425.

21. Miyazaki H. Oral malodor in the general population of Japan. Bad breath: research perspectives. 1995; 119-136.

22. Frexinos J, Denis P, Allemand H, Allouche S, Los F, Bonnelye G. Descriptive study of digestive functional symptoms in the French general population. Gastroenterologie clinique et biologique. 1998; 22(10): 785-91.
23. Söder B, Johansson B, Söder P. The relation between foetor ex ore, oral hygiene and periodontal disease. Swed. Dent. J. 2000; 24(3): 73-82.

24. Sanz M, Roldan S, Herrera D. Fundamentals of breath malodour. J. Contemp. Dent. Pract. 2001; 2(4): 1-17. https:// doi.org/10.5005/jcdp-2-4-22.

25. Al-Otaibi M. Themiswak (chewing stick) and oral health. Studies on oral hygiene practices of urban Saudi Arabians. Swed. Dent. J. Suppl. 2004; 167: 2-75.

26. The detection and measurement of oral malodor in periodontitis patients [internet]. http://52.172.27.147:8080/ jspui/handle/123456789/461. Date accessed: 2005.

27. Miyazaki H, Sakao S, Katoh Y, Takehara T. Correlation between volatile sulphur compounds and certain oral health measurements in the general population. J. Periodontol. 1995; 66(8): 679-684. https://doi.org/10.1902/ jop.1995.66.8.679.

28. Morita M, Wang HL. Relationship between sulcular sulfidelevel and oral malodor in subjects with periodontal disease. J. Periodontol. 2001; 72(1): 79-84. https://doi.org/10.1902/ jop.2001.72.1.79.

29. Kishi M, Kimura S, Yuko ON, Kishi K, Aizawa F, Moriya T. Oral malodor and periodontopathic microorganisms in tongue coat of periodontally healthy subjects. Dentistry in Japan. 2002; 38: 24-28.

30. Almas K, Al-Sanawi E, Al-Shahrani B. The effect of tongue scraper on mutans streptococci and lactobacilli in patients with caries and periodontal disease. Odonto-stomatologie tropicale $=$ Trop. Dent. J. 2005; 28, 109: 5-10.

31. Signoretto C, Burlacchini G, Bianchi F, Cavalleri G, Canepari P. Differences in microbiological composition of saliva and dental plaque in subjects with different drinking habits. The new microbiologica. 2006; 29(4): 293-302. 